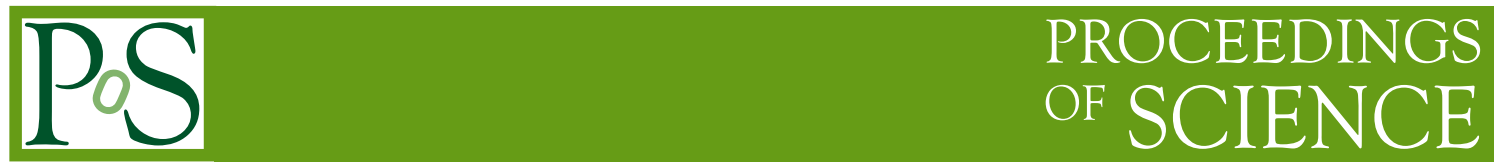

\title{
The LHCb upgrade detector
}

\section{Burkhard SCHMIDT*†}

CERN, PH-department, CH-1211 Geneva 23

E-mail: Burkhard.Schmidtecern.ch

The LHCb experiment has been designed to perform precision measurements of CP asymmetries and to investigate potential effects of physics beyond the Standard Model. Results obtained from data collected in 2010 and 2011 show that the detector is robust and functioning well. While $\mathrm{LHCb}$ will be able to measure a host of interesting channels in heavy flavour decays in the coming years, a limit of about $1-2 \mathrm{fb}^{-1}$ of data per year can only be overcome by upgrading the detector. With a detector read out at $40 \mathrm{MHz}$, a much more flexible software-based triggering strategy will allow a large increase not only in the data rate, as the detector will collect $5 \mathrm{fb}^{-1}$ per year, but also in the ability to increase trigger efficiencies especially in decays to hadronic final states. In addition, it will be possible to modify trigger algorithms in order to explore different physics as LHC discoveries point to the most interesting channels. Here a short description of the physics motivations and proposed detector changes for exploring new phenomena in proton-proton collisions near $14 \mathrm{TeV}$ centre-of-mass energy are presented.

XXIst International Europhysics Conference on High Energy Physics

21-27 July 2011

Grenoble, Rhônes Alpes France

${ }^{*}$ Speaker.

$\dagger$ on behalf of the LHCb collaboration 


\section{Introduction}

The main purpose of $\mathrm{LHCb}$ is to discover and determine the properties of physics beyond the Standard Model (SM). This is a powerful approach because decays of $b$ and $c$ quarks can probe large mass scales, beyond that reachable by direct new particle production, via virtual quantum loops.

Given that the $b \bar{b}$ pairs are predominantly produced in the forward or backward direction, the $\mathrm{LHCb}$ detector was designed as a forward spectrometer, covering a pseudo-rapidity range of $1.9<\eta<4.9$. The detector elements are placed along the beamline of the LHC, as shown in Fig. 1 [1]. The Vertex Locator (VELO), a silicon strip device, surrounds the proton-proton interaction region and provides excellent impact parameter and proper time resolutions. In conjunction with the VELO, the main devices used to measure track momenta comprise a large area silicon strip detector (TT) located in front of a 4 Tm dipole magnet, and a combination of silicon strip detectors and straw drift chambers (T1-T3) placed behind. Two ring-imaging Cherenkov (RICH) detectors are used to identify charged hadrons. Further downstream an Electromagnetic Calorimeter (ECAL) is used for photon detection and electron identification, followed by a Hadron Calorimeter (HCAL), and a Muon system consisting of alternating layers of iron and chambers (MWPC) to distinguish muons from hadrons. The ECAL, HCAL and Muon System provide the capability of first-level hardware triggering.

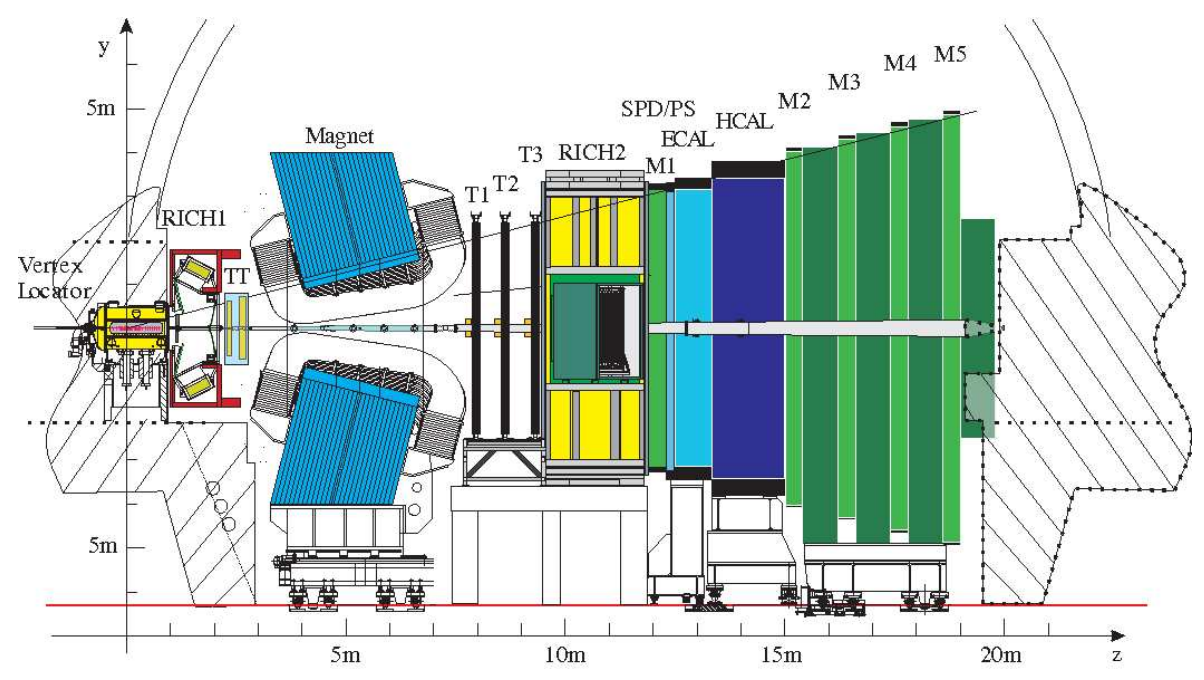

Figure 1: Layout of the $\mathrm{LHCb}$ detector.

During 2011 the experiment ran at an instantaneous luminosity of about $3.5 \times 10^{32} \mathrm{~cm}^{-2} \mathrm{~s}^{-1}$ and an average number of interactions per crossing of $\mu \sim 1.5$. This rate is about twice the original planned instantaneous luminosity and four times the expected $\mu$. The detector operated very well under these conditions [2]. In 2011 LHCb has accumulated $1.1 \mathrm{fb}^{-1}$ of data and expects to collect at least $5 \mathrm{fb}^{-1}$ before the second long LHC shutdown in 2018. The collaboration is planning to upgrade the detector in 2018 in order to run the experiment in the following years at luminosities of up to $2 \times 10^{33} \mathrm{~cm}^{-2} \mathrm{~s}^{-1}$ and with a substantial increase of trigger efficiency for hadronic final states. The upgraded detector will be able to collect $50 \mathrm{fb}^{-1}$ of data integrated over around ten years of operation [3]. 


\section{Physics goals of the current and upgraded experiment}

A particular attraction of performing flavour physics at the LHC is the opportunity to make measurements of $C P$-violating asymmetries with much higher precision than has been possible hitherto. These asymmetries are very sensitive to the contribution of physics beyond the Standard Model. A particular focus of this programme is on the $B_{s}$ sector, about which very little is known and where new physics effects may be apparent. LHCb and its upgrade offer unique possibilities to enhance our knowledge of flavour physics to unprecedented levels.

The LHCb physics programme will be executed in two phases. In the first phase of the experiment, i.e. with data collected up to 2017 with the current detector, LHCb will obtain results that will severely test the SM. It will be possible to extend significantly the precision of many key parameters in $B$ and $D$ physics beyond what was possible at the B-factories, and make the first exploration of the $B_{s}$ system. The hope is to observe thorough non-Standard Model effects. Whatever the outcome of this initial exploration, however, precision measurements of key parameters will be required. They will be carried out in the second phase of the experiment with the upgraded detector. An improved knowledge of the key parameters and observables will be essential to understand the physics beyond the SM, which hopefully will be uncovered at the LHC.

The scientific goals of LHCb extend also beyond quark-flavour physics. Important studies are possible in the lepton sector, including the search for lepton-flavour violating tau decays and for low mass Majorana neutrinos. Furthermore, the forward geometry, precise vertexing and particle identification capabilities of the detector give LHCb unique and exciting possibilities in the areas as diverse as electroweak physics, the search for long-lived new particles, and QCD. In all cases great benefit will come both from the increased sample sizes that will be made available with the upgrade, and the flexible software trigger. Therefore, the upgraded LHCb experiment can be regarded as a general-purpose detector in the forward direction.

\section{The LHCb Trigger upgrade}

The present first level trigger (L0) is implemented in hardware [1]. Trigger selections are made at the $40 \mathrm{MHz}$ beam crossing rate using either the Calorimeters or the Muon System. The detector is read-out at a maximum rate of $1 \mathrm{MHz}$. To trigger at an increased event rate requires a substantial change in the $\mathrm{LHCb}$ read-out architecture.

The criteria for the present L0-triggers are based on the deposit of several GeV of transverse energy, $E_{\mathrm{T}}$. While this provides high efficiencies on dimuon events, fully hadronic signal decays typically have an efficiency less than $50 \%$. In these hadronic decays the $E_{\mathrm{T}}$ threshold required to reduce the rate of triggered events to an acceptable level is already a substantial fraction of the $B$ meson mass. Any further increase in the rate requires an increase of this threshold, which then removes a substantial fraction of signal decays. The trigger yield therefore saturates for hadronic channels with increasing luminosity.

To overcome this situation it is essential to remove the $1 \mathrm{MHz}$ L0 limitation and to introduce information in the trigger that is more discriminating than $E_{\mathrm{T}}$, e.g. displaced vertex information. The most effective way of achieving this is to supply the full event information at the $40 \mathrm{MHz}$ beam crossing frequency and to analyze each event in a trigger system implemented in software. A de- 
tector upgraded in this way would allow the yield of hadronic $B$ decays to be increased significantly for the same LHC machine run-time.

\section{The LHCb detector upgrade}

A new electronics architecture is necessary to satisfy the requirements of reading data from every bunch-crossing in the upgraded LHCb. Many of the challenges of the $40 \mathrm{MHz}$ readout scheme can be met using modern technologies adapted for high energy physics. For example, high-speed optical links will be installed to accommodate the increase in data volume from the detector. Data compression schemes will be implemented on the detectors to reduce the number of these links. In order to minimise cost, development time and installation effort, parts the existing electronics that satisfies the upgrade requirements will be re-used and common devices and modules will be developed. A detailed overview of the upgraded detector is given in reference [3].

The physics programme of the LHCb upgrade requires an extremely performant vertex detector with fast pattern recognition capabilities, very good vertex resolution and two track separation. Sufficient radiation hardness is important to guarantee excellent performance throughout the upgrade data-taking period. As the VELO detector is very near to the beamline, its material budget should be as light as possible to increase vertexing and tracking performance. Moreover, because the trigger performance of the upgrade relies heavily on vertex detector data, its use in the trigger must be fast and flexible enough to adapt to the evolving physics needs of the experiment.

Another main challenge of the LHCb upgrade lies in the redesign of the tracking system, which should be able to sustain luminosities up to $2 \times 10^{33} \mathrm{~cm}^{-2} \mathrm{~s}^{-1}$. The new tracking systems which will replace the TT and parts of tracking stations T1-T3, are based on two possible options: one relies on fibers readout by silicon photo-multipliers, and the other on silicon strips. As for the VELO, the tracking detectors must ensure radiation resistance, good granularity, low material budget and fast response to minimize spillover events in the $25 \mathrm{~ns} \mathrm{LHC}$ bunch scheme.

The particle identification (PID) system is also a vital component of the upgraded detector. Several key physics channels which involve kaons rely on the RICH PID to reject copious backgrounds from multiple-track combinatorics and events with similar decay topologies. The current RICH system employs custom-built Hybrid Photon Detectors (HPD), the Pixel HPDs [1], which operate very successfully. However these cannot be re-used in the upgraded RICH detector since the HPD readout electronics are limited to a $1 \mathrm{MHz}$ event readout rate, incompatible with the upgrade rate of $40 \mathrm{MHz}$. It is therefore proposed to replace the HPDs with Multi-anode Photomultipliers (MaPMTs) with external $40 \mathrm{MHz}$ readout electronics. The aerogel system in RICH1 will be eliminated and could be replaced by another particle ID device based on time of flight that uses Cherenkov light in quartz, called the TORCH, complementing the RICH detectors in the lowmomentum particle ID. The TORCH is a challenging project, and its installation could come later, without compromising the initial operation of the upgraded detector.

Coping with luminosities of $10^{33} \mathrm{~cm}^{-2} \mathrm{~s}^{-1}$ does not require substantial rebuilds of the Calorimeter and Muon systems. It is planned for the upgrade to keep the present ECAL and HCAL calorimeter modules, their photomultipliers and Cockroft Walton bases. However, to keep the same average anode current of the phototubes at the higher luminosity, their high voltage is reduced and therefore the gain of the amplifier integrator in the Front-End card will be increased. 
The muon system Front-End electronics is already read out at $40 \mathrm{MHz}$, as it is currently sending data at this rate to the L0 trigger. Only small modifications are needed to have the system fully integrated with the rest of the upgraded DAQ.

Finally, more disk storage and more computing power will be needed to cope with a factor 10 more events at the output of the HLT. The upgraded detector will be able to collect at least $5 \mathrm{fb}^{-1}$ per year, running at a luminosity of up to $2 \times 10^{33} \mathrm{~cm}^{-2} \mathrm{~s}^{-1}$. If one considers the increase in trigger efficiency for the hadronic channels, this will result in a yield of events at least ten times greater than the present experiment.

\section{Conclusions and Outlook}

The upgraded LHCb detector will allow precision measurements to be made, with good prospects for discovering physics beyond the Standard Model. In addition, other physics in the forward direction at the LHC can be pursued. The upgraded LHCb detector will allow an order of magnitude more data to be collected than at the current experiment. The upgrade is being organized to be ready for data-taking after the second long shutdown of the LHC, which is scheduled for 2018.

\section{References}

[1] The LHCb Collaboration, The LHCb Detector at the LHC, 2008 JINST 3 S08005.

[2] M.G. van Beuzekom, Performance of the LHCb Detector during 2010-2011 data taking, these proceedings.

[3] The LHCb Collaboration, Letter of Intent for the LHCb Upgrade, March 2011, CERN-LHCC-2011-001 